\title{
Three-dimensional organization of electrical turbulence in the heart
}

\author{
A. V. Panfilov \\ Department of Theoretical Biology, University of Utrecht, Padualaan 8, 3584 CH Utrecht, The Netherlands
}

(Received 18 December 1998)

\begin{abstract}
Three-dimensional organization of electrical turbulence that is induced via the phenomenon of spiral breakup is studied in a computer model of the heart, which includes realistic ventricular geometries and cardiac anisotropy. We find filaments of rotors during the turbulence and study their number and length as a function of the heart size. We perform a dimensional analysis of turbulence. [S1063-651X(99)50606-5]
\end{abstract}

PACS number(s): 47.65.+a, 82.40.Fp, 05.45.-a, 87.19.Rr

Electrical turbulence in the heart results in ventricular fibrillation, which is the main cause of sudden cardiac death in the industrialized world [1]. Although heart rhythm during turbulence is irregular, recent experimental data show a large amount of spatial and temporal organization [2-6,1]. High resolution optical recordings of electrical activity during turbulence show that electrical turbulence in the heart consists of many "elementary" sources of excitation which can be rotors $[7,8,5,6]$, or more general sources which were called "phase singularities" [5]. A rotor is a circulation of the wave around a point, or a small region in two dimensions. Rotors occur in systems that are called excitable, i.e., systems that have two main properties: excitability (ability to conduct waves of excitation) and refractoriness (ability to recover and conduct another wave of excitation after some period of time called the refractory period). Such rotors were found in cardiac tissue $[7,8]$ as well as in several other biological, physical, and chemical systems [9], and in many simplified models of cardiac tissue [10]. "Phase singularities" can be viewed as surface manifestations of a threedimensional (3D) version of a rotor. If circulation of the wave occurs in a 3D medium, then the region in 3D space around which the wave circulates is a "singular filament." The points of exit of this "singular filament" on the surface give the points of "phase singularities" [9].

After the elementary units of turbulence in the heart were found, the next important step is to quantify the observed picture, and the main questions are: How many "singular filaments" organize the turbulence? What is the length of these filaments? How do they depend on parameters? Unfortunately it is extremely difficult to answer these questions in experimental studies because the wave propagation in the heart is three dimensional, while experimental pictures, at the moment, can be recorded only from the epicardial surface of the heart.

In this paper we make the first attempt to quantify the overall 3D pattern of turbulence in an anatomical model of the heart using the method of numerical experiment. The model of turbulence in this study is based on the phenomenon of spiral breakup [11-16]. The phenomenon of spiral breakup involves the degeneration of one or more rotors into a complicated spatiotemporal pattern in a homogeneous excitable media due to some kind of instability. Spiral breakup occurs in many models of excitable media including models of cardiac tissue [11,12]. Recent studies have shown that turbulence induced via the phenomenon of spiral breakup resembles turbulence recorded experimentally in the heart [4]. The anatomical model of heart [17] is based on extensive experimental measurements of the heart structure [18] and includes realistic ventricular geometries and cardiac anisotropy. Since one of the most fundamental properties of electrical turbulence in the heart is its dependence on the heart size [19,5], we studied how main characteristics of "singular filaments" depend on the size of the heart.

The spread of excitation in the heart was governed by a conventional parabolic partial differential equation:

$$
C_{m} \partial e / \partial t=\operatorname{div}(D \nabla e)+I_{m},
$$

where $D$ is the conductivity tensor, $e$ is the transmembrane potential and $I_{m}$ is the total transmembrane ionic current per unit area [20].

The transmembrane current was described using a simplified excitable dynamics equations of the FitzHugh-Nagumo type, published earlier [21]:

$$
\begin{gathered}
I_{m}=-k e(e-a)(e-1)-e g, \\
\partial g / \partial t=[\epsilon+(\mu 1 g) /(\mu 2+e)][-g-k e(e-a-1)] .
\end{gathered}
$$

This description reproduces overall characteristics of cardiac tissue such as refractoriness, dispersion relation, rate duration properties, etc. The particular parameters in this model, $\epsilon, \mu 1, \mu 2$, etc., do not have a clear physiological meaning, but are adjusted to reproduce these overall characteristics of cardiac tissue. The parameters of the model in a breakup regime were the following: $a=0.1, \mu 1=0.07 ; \mu 2$ $=0.3, k=8, \boldsymbol{\epsilon}=0.01$. The conductivity tensor was computed from the fiber orientation field using the method published in [17]. The tissue anisotropy was chosen to be $1: 2$ with respect to the velocity of wave propagation; the value of $C_{m}$ was $C_{m}=1$. Equations (1) and (2) were numerically integrated in a domain representing the heart shape using the Euler method with Neumann boundary conditions. The wavelength of reentry was $40 \mathrm{~mm}$. (The wavelength here is the product of the average temporal period during the turbulence by the speed of the wave). To initiate turbulence we set up standard initial conditions in a form of a single wave break located either in a free wall of the left ventricle or in a free wall of the right ventricle [17]. This wave break first initiates a rotating spiral wave that, after several rotations, breaks into a 

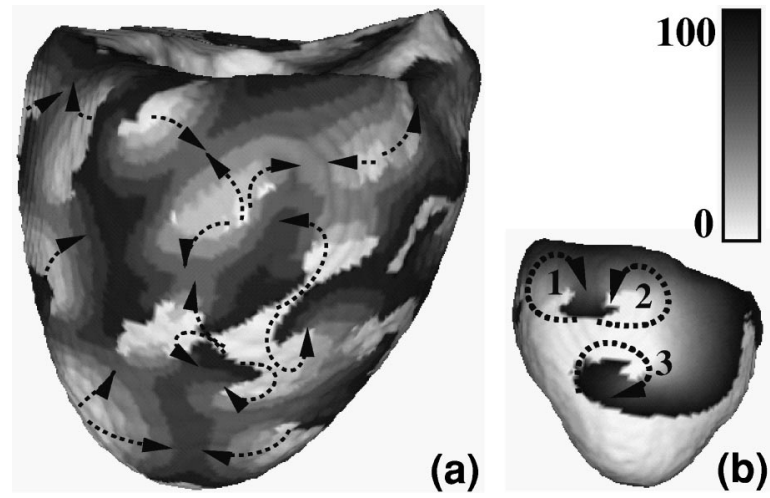

FIG. 1. Rendering of the activation map on the surface of the heart during electrical turbulence due to the process of spiral breakup. The coding of the activation time in ms is shown by a legend bar. (a) The size of the heart measured from apex to base is $94 \mathrm{~mm}$ and (b) the size of the heart is $51.7 \mathrm{~mm}$.

turbulent regime. The patterns of excitation during the turbulence regime, obtained from different initial conditions, were similar.

We made computations for the hearts of six different sizes ranging from $94 \mathrm{~mm}$ to $51.7 \mathrm{~mm}$. The size of the heart here is just the distance from the top point of the heart (base) to the bottom point of the heart (apex). The hearts of different sizes were obtained by scaling the same set of anatomical data [18] into cubes of different sizes in the following way. The basic data set [18], which includes the 94-mm heart, was represented as voxels in the $129 \times 129 \times 129$ cube. In order to obtain the other hearts, we just scaled these data to cubes of other sizes. For example, in order to obtain the $51.7-\mathrm{mm}$ heart, we scaled the original data from the cube of 129 $\times 129 \times 129$ elements to the cube of $71 \times 71 \times 71$ elements. Therefore, all of the hearts considered in this paper are geometrically similar, i.e., the 94-mm heart compared to the 51.7-mm heart has $94 / 51.7=1.8$ times thicker myocardial walls, $1.8^{2}$ larger surface, and $1.8^{3}$ larger volume.

Figure 1 shows two typical surface patterns of excitation that occur during turbulence in our model. Here color codes the location of the front of an excitation wave at different moments in time. White color shows the front position at some initial zero moment of time, while the black color shows the front position at $100 \mathrm{msec}$. Arrows show the main path of excitation. The pattern that occurs in the small heart is relatively simple: we can see two closely counter rotating rotors (numbers 1 and 2) that, in physiological literature, are called "figure-of-eight reentry." There is also a single rotor (number 3 ) in the middle of the heart surface. In a large heart the patterns of excitation are not so apparent. Although we can see regions of spatially correlated wave propagation, the sources underlying such waves are not apparent. These patterns are similar to those observed in experimental recordings $[5,6]$.

The spatiotemporal dynamics of excitation resembles the dynamics described in Refs. [5,6]. The dynamics in the small heart is quasi-two-dimensional. We can nearly always see rotating and drifting rotors on the surface of the heart. This dynamics is similar to the experimentally recorded dynamics [5] and to the dynamics of the initial phase of fibrillation [6]. The dynamics of excitation in the large heart is three dimen-
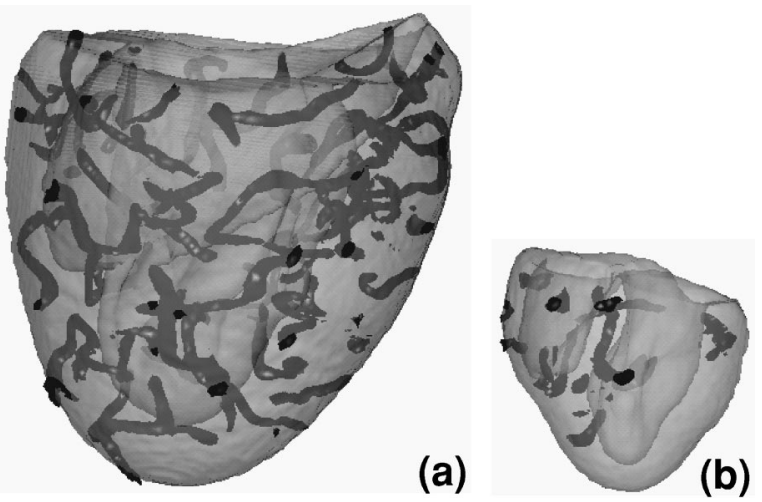

FIG. 2. Filaments in the heart during turbulence. (a) The size of the heart is $94 \mathrm{~mm}$ and (b) the size of the heart is $51.7 \mathrm{~mm}$.

sional and resembles the dynamics of chronic fibrillation [6]. There are no persistent rotors on the surface of the heart and, usually, a considerable amount of excitation comes to the surface from the subsurface midmyocardial region.

Now let us consider the 3D organization of these wave patterns. As we mentioned above, the elementary unit of turbulence in a 3D excitable medium is a "singular filament', [9]. In order to find it, note that each rotor in Fig. 1(b) has a small region around which the spiral rotates. [In Fig. 1(b) they are approximately located at the positions of numbers 1,2 , and 3.] If we extend these regions to the midmiocardium, we will get cylindrical tubes around which the wave rotates in three dimensions. These tubes will be "singular filaments.' The use of filaments substantially reduces the amount of information necessary to depict the complex 3D spatiotemporal pattern of fibrillation. Figure 2 shows filaments found for patterns of excitation from Fig. 1. We see a large number of filaments (about 50) in the 94-mm heart. For the $51.7-\mathrm{mm}$ heart, the number of filaments is substantially less; in Fig. 2(b) we can find only about 10 filaments. If we compare Fig. 1(b) with Fig. 2(b) we see that the two rotors (2 and 3) are generated by a single filament and, in fact, they represent just one source of excitation. We can also see that the left-upper wave break is a part of a short filament extending to the endocardium of the right ventricle. A comparison of Figs. 1(a) and 2(a) is much more difficult because there are so many filaments. We can still find a correspondence between the individual filaments and surface dynamics. However, we see that large portions of filaments and even whole filaments are located beneath the surface of the heart. Apparently not all of them are manifested on the surface. These "latent" filaments that cannot be seen on the surface may play an important role in the dynamics of the patterns of excitation because all filaments interact. This can result in complex dynamics [22]. Note that some of the filaments in Fig. 2 look as if they stick out of the heart surface, which is just an artifact of the graphical program used.

We determined the average characteristics of filaments found after analyzing 600 patterns of excitation in the hearts of six different sizes (Fig. 3). The patterns of excitation that were obtained form four different initial conditions. The time interval between two successive patterns of excitations was more than $1500 \mathrm{msec}$. For the 94-mm heart the average number of filaments is $38 \pm 4.85$. The average number of filaments rapidly decreases with decreasing heart size; for the 


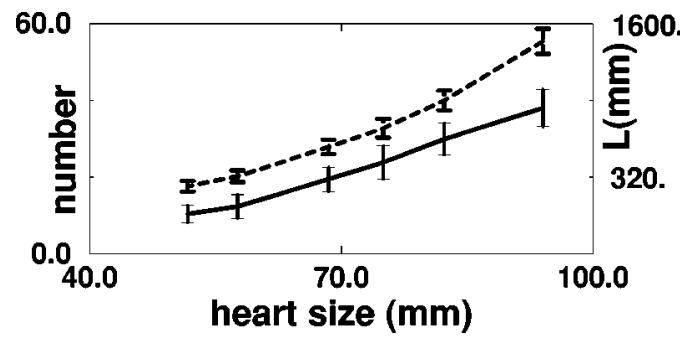

FIG. 3. Total number of filaments (solid line) and the total length of filaments in $\mathrm{mm}$ (dashed line) vs heart size ( $\mathrm{mm}$ ). The left vertical axis $n$ is for the total number of filaments, the right vertical axis $L$ is for the total length of filaments.

51-mm heart the number of filaments is only 10.4 \pm 2.2 . We also determined the total length of all filaments in the heart (Fig. 3). For the 94-mm heart the total length of all filaments is $1450 \pm 106 \mathrm{~mm}$, which is about 15 times longer than the distance between the base and apex. When the size of the heart decreases the total filament length decreases rapidly; in the $51-\mathrm{mm}$ heart it is about $243 \pm 43 \mathrm{~mm}$, which is only five times the heart size.

We have also computed the average length of a filament in the hearts of different sizes. In Fig. 4 we plotted the ratio of this average filament length to the typical thickness of a myocardial wall. (In our model the typical thickness of a myocardial wall was about 0.17 of the heart size, e.g., for the 94-mm heart it was about $16 \mathrm{~mm}$.) We see (Fig. 4) that, in hearts of all sizes, the average filament is about 2.5 longer than the typical wall thickness. This shows that a "regular" filament is not a straight transmural line but is curved. Such elongation maybe a result of a twist induced instability of filaments in a rotationally anisotropic media reported in Refs. $[23,24]$.

To better understand the dependence of electrical turbulence on heart size, we performed a dimensional analysis for two characteristics of turbulence in the heart: the total number of filaments in the heart at a given moment of time and the total length of all filaments comprising the turbulence. The results of such scaling are shown in Fig. 5. The solid line in Fig. 5 shows estimates for the ln of the average total length of all filaments during the turbulence vs the ln of the heart size. We see that the points can be approximated satisfactorily by a straight line. The slope of this line is 2.98 \pm 0.025 . The dashed line in Fig. 5 shows the $\ln$ of the average number of filaments during the turbulence vs the ln of the heart size. The slope of this line is $2.21 \pm 0.034$.

The results of this scaling can be interpreted as follows. The slope $2.98 \pm 0.025$ indicates that the turbulence in the heart is indeed three dimensional; the total length of all filaments increases with the volume of the heart in such a way

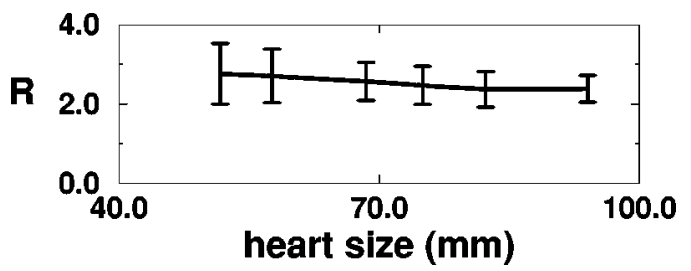

FIG. 4. Ratio of the average filament length to the typical thickness of a myocardial wall $(R)$ vs heart size $(\mathrm{mm})$.

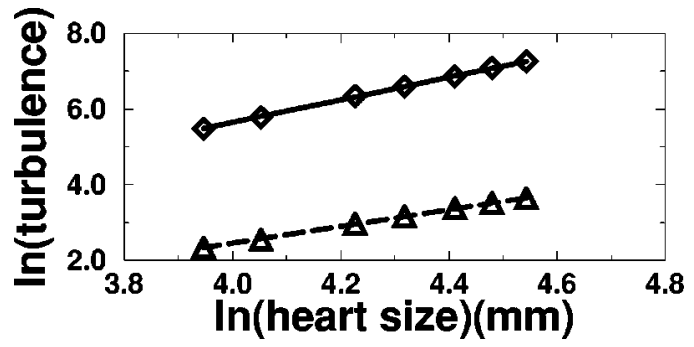

FIG. 5. Scaling of ventricular turbulence. The $\ln$ of the total length is shown by the solid line; the ln of the number of filaments is shown by the dashed line.

that the filament length per unit volume remains constant. The slope for the line of the total number of filaments 2.21 \pm 0.034 shows that the number of filaments is not directly proportional to the volume of the heart. This slope, in fact, is close to 2, which indicates that the total number of filaments in the heart is approximately proportional to the area of the heart surface.

Therefore we can conclude that electrical turbulence in the heart, based on the phenomenon of spiral breakup, is a complex 3D process, which involves many sources of excitation (about 30 or more in a large heart); the total filament length of the sources that organizes turbulence far exceeds the characteristic heart size. The number of sources during the turbulence is approximately proportional to the area of the heart surface.

Finally, we studied possible effects of space discretization on the results of our computations. For that we studied turbulence in the $94 \times 94 \times 94$ voxel heart for different values of the space steps: $0.9,0.8$, and 0.7 from the value of the space step used in previous simulations. We did not find any significant differences from our previous results. For example, the average number of filaments for the 0.8 space step was $12.8 \pm 3.1$ (the heart size is 54.8 ). This is very close to the extrapolated average number of filaments (11.3) found in previous computations for a heart of same size (Fig. 3). Note that, due to the large standard deviation, the difference in the number of filaments is not conclusive. However, the larger number of filaments at a smaller space step may indicate that the minimal distance between rotors becomes smaller at smaller space steps. Similar results were obtained for other values of space steps.

Our model lacks several important features of the heart and circulatory system, such as heart mechanics, neurophysiology, baroreceptors, etc. However, many of these features are not important for comparison with experiments $[5,6]$ because these experiments were performed on isolated hearts with blocked mechanical activity. Note that our study was focused on ventricular fibrillation and, therefore, our heart model did not include atria, AV and SA nodes.

In computations reported in this paper, the heart size varied from $94 \mathrm{~mm}$ to $51 \mathrm{~mm}$. However, these sizes were computed for the wavelength of $40 \mathrm{~mm}$. If the value of the wavelength is different, then the heart size will have to be recomputed. The easy way to do this is to measure heart sizes in dimensionless units: size $=S / \lambda$, where $S$ is the actual size of the heart from base to apex, and $\lambda$ is the characteristic 
wavelength of a rotor in the heart. In such dimensionless units the size of the heart in Fig. 1(a) is 2.4 units, and in Fig. $1(b)$ is 1.2 units.

Note that the dimensionless heart size $($ size $=S / \lambda)$ changes with a change in wavelength, even if the actual heart size is constant. Therefore, the dimension analysis performed in this paper can, in some cases, be applied to changes that occur due to changes in the wavelength of rotors in a heart of the same size. Using this fact, we can assume that the transition to chronic "three-dimensional" turbulence reported in
[6] was due to a decrease in the wavelength of rotors in the course of time. [Decreasing the wavelength is equivalent to increasing the heart size, which can make patterns of excitation three dimensional, as demonstrated in Figs. 1(b) and 1(a).]

We are grateful to Professor P. Hogeweg for valuable discussions and help with this research. We wish to thank S. M. McNab for linguistic advice.
[1] A. V. Holden, Nature (London) 392, 20 (1998).

[2] R. A. Gray et al., Science 270, 1222 (1995).

[3] F. X. Witkowsky et al., Phys. Rev. Lett. 75, 1230 (1995).

[4] A. Garfinkel et al., J. Clin. Invest. 99, 305 (1997).

[5] R. A. Gray, A. M. Pertsov, and J. Jalife, Nature (London) 392, 75 (1998).

[6] F. X. Witkowsky et al., Nature (London) 392, 78 (1998).

[7] M. A. Allessie, F. I. M. Bonke, and F. J. G. Schopman, Circ. Res. 33, 54 (1973).

[8] A. M. Davidenko, J. M. Pertsov, R. Salomontsz, W. Baxter, and J. Jalife, Nature (London) 355, 349 (1991).

[9] A. T. Winfree and S. H. Strogatz, Nature (London) 311, 611 (1984).

[10] A. T. Winfree, Science 266, 1003 (1994).

[11] A. T. Winfree, J. Theor. Biol. 138, 353 (1989).

[12] A. V. Panfilov and A. V. Holden, Phys. Lett. A 147, 463 (1990).

[13] H. Ito and L. Glass, Phys. Rev. Lett. 66, 671 (1991).
[14] A. Karma, Phys. Rev. Lett. 71, 1103 (1993).

[15] M. Courtemanche, L. Glass, and J. P. Keener, Phys. Rev. Lett. 70, 2182 (1993).

[16] A. V. Panfilov and P. Hogeweg, Science 270, 1223 (1995).

[17] A. V. Panfilov, in Computational Biology of the Heart, edited by A. V. Panfilov and A. V. Holden (Wiley, Chichester, 1997), pp. 259-276.

[18] P. J. Hunter, B. H. Smail, P. M. F. Nielson, and I. J. LeGrice, in Computational Biology of the Heart (Ref. [17]), pp. 171215.

[19] W. E. Garrey, Am. J. Physiol. 33, 397 (1914).

[20] A. V. Holden and A. V. Panfilov, in Computational Biology of the Heart (Ref. [17]), pp. 65-99.

[21] R. R. Aliev and A. V. Panfilov, Chaos Solitons Fractals 7, 293 (1996).

[22] A. T. Winfree, Nature (London) 371, 233 (1994).

[23] F. Fenton and A. Karma, Chaos 8, 20 (1998).

[24] F. Fenton and A. Karma, Phys. Rev. Lett. 81, 481 (1998). 